transit. The peptidergic neurone as a model system was the dominating theme of the recent 8 th International Symposium on Neurosecretion*. Also at this meeting, H.-D. Dellman (Iowa) showed that, in the developing neurone, secretory material appears within the cisternae of the endoplamic reticulum before the appearance of Golgi complexes or secretory granules. It is tempting to relate this observation to the almost simultaneous report of A. G. Robinson (Pittsburgh), at another meeting $\dagger$, that while immunoreactive neurophysin appeared in the brains of fetal

*Held at Friday Harbor, Washington on 4-10 September 1980. rats on day 13 of gestation, peaked on day 14 and fell slowly towards term, the hormones were undetectable on day 13 but their content in the brain increased towards term. If, as Robinson suggests, these results reflect a high relative concentration of precursor forms in the third fetal week, then, taken together with Dellman's findings, they suggest that the developing hypothalamo-neurohypophysial system presents a tool for the study of the role of the Golgi complex in precursor processing.

†'Neuropeptides, development and ageing'; held at Rochester, New York on 3-5 September 1980.

\title{
The search for the Zvolen fireball
}

from David W. Hughes

Five all-sky camera stations of the European Fireball Network recorded a bright fireball which penetrated low into the atmosphere on 27 May 1979 at $20 \mathrm{~h}$ $39 \mathrm{~min}$. The fireball terminated close to Zvolen in central Slovakia and may have produced several meteorites.

Geometric, dynamic, photometric and orbital data have been calculated by $Z$. Ceplecha, J. Boček and M. Ježková of the Astronomical Institute of the Czechoslovak Academy of Sciences, Ondfejov, V. Porubcăn of the Astronomical Institute of the Slovak Academy of Sciences, Bratislava and G. Polnitzky of the University Observatory, Vienna. Their results have been published in a recent edition of the Bulletin of the Astronomical Institutes of Czechoslovakia

\section{1, 176; 1980.}

The photometric and positional data on all the available photographic records were measured using a Zeiss Ascorecord device. Computational analysis gave the light curve shown in Fig. 1. It is interesting to note that the magnitude remained reasonably constant during a considerable portion of the trajectory. The luminous power is approximately proportional to the kinetic energy lost per unit time by the incident body and it can be seen that mass loss dominates the constant luminosity phase. The maximum deceleration occurs well inside the luminous trajectory and this is typical for meteorite-dropping fireballs. The end height, coupled with the initial mass, velocity and trajectory, indicates that the meteorite was an ordinary

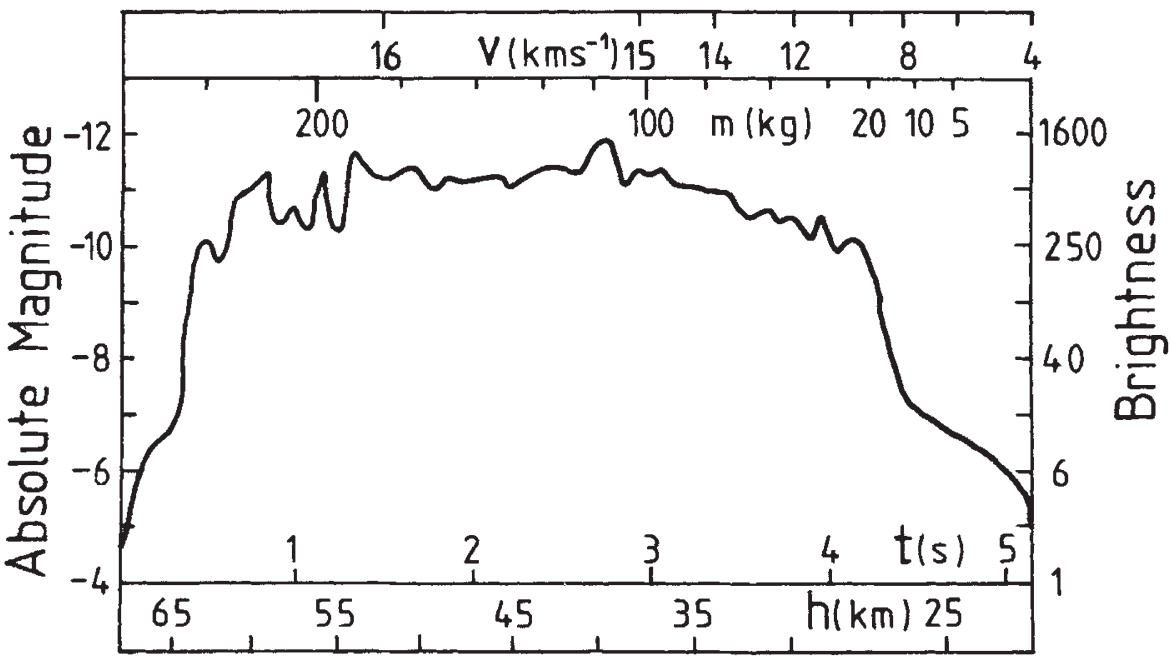

Fig. 1 The light curve of the Zvolen Fireball over the brightest part of its trajectory. The left ordinate gives the absolute photometric magnitude, which is the magnitude the fireball would have if it was at a standard position $100 \mathrm{~km}$ from the observer at the zenith. The right-hand ordinate gives the brightness, standardized so that a brightness of 1 arbitrary unit is equivalent to an absolute magnitude of -4 . The main abscissa is time, measured in seconds. Secondary abscissae show the decrease in the height of the causative meteorite and also its mass loss and deceleration. At $t=0$, the meteorite had a calculated mass of $223 \mathrm{~kg}$ and a velocity of $16.12 \mathrm{~km} \mathrm{~s}^{-1}$.

chondrite (a 'strong' stone). The low initial velocity is responsible for the maximum luminosity occurring between heights of 60 and $30 \mathrm{~km}$. Two of the photographs showed the separation of three fragments from the main trail. Fragmentation occurred at heights of 28,33 and $40 \mathrm{~km}$.

The computational analysis also gave the orbit of the incident meteorite. This had a perihelion distance of $0.89 \mathrm{AU}$ (just inside the Earth's orbit), an aphelion distance of $2.84 \mathrm{AU}$ (right in the asteroid beit) and an inclination to the ecliptic of only $2.3^{\circ}$.

The mass of the final falling meteorite and fragments was estimated to be a kilogram or so. The statistics of meteoritic events from fireball camera networks in the American and Canadian Prairies, demonstrate that one observable meteorite fall per year should occur within a network area of about $1.4 \times 10^{6} \mathrm{~km}^{2}$. As the cameras can only register fireballs efficiently when the sky is completely dark, the influx rate of meteorites will be about three times higher than this figure. This is equivalent to about 1,100 meteorite falls per year over the whole surface of the Earth and about 300 over the land masses. In the regions where the networks are active it has been found that the searches that follow the observed falls only have, on average, a 10 per cent chance of recovering the meteorite. Under these assumptions, the Slovakian part of the European network should observe a meteorite fall every 30 years and recover one every 300 years.

The search area for the Zvolen meteorite was estimated to be about $10 \mathrm{~km}^{2}$. This is larger than usual. The photographic station which predicts the fall site most accurately is the one closest to the terminal point of the luminous trail. The camera at Močiar was only $15 \mathrm{~km}$ from the predicted position but the gremlins that haunt all scientific endeavour were at work. The Močiar camera was opened on that evening two minutes after the apparition of the fireball. The central part of the search area lies in the eastern part of the town of Zvolen, a region containing many urban dwellings, two large factories, a railway depot with piles of coal and a small river that is swampy in parts. The other regions of the predicted fall area are hilly and covered with woods, fields and meadows. The possibilities of finding a strange, $1-\mathrm{kg}$ rock, about $10-\mathrm{cm}$ across, do not seem too great.

Great publicity was given to the event in the hope of obtaining eye-witness reports of the fireball flight near the impact point. It was surprising that only seven reports were forthcoming in a region of such dense population and only two reports helped to reconstruct the whole path of the flight.

Well over a year has passed, including an early spring when vegetation is much thinner than usual. Nothing has yet been found.

David $W$. Hughes is in the Department of Physics, University of Sheffield. 